\title{
JOURNAL.RU
}

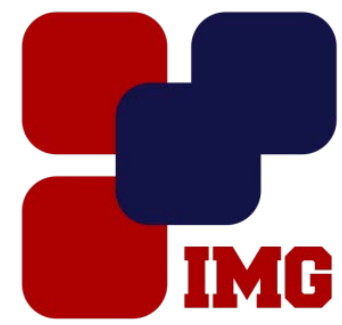
IYANOY Management GROUP

Бадриев И.Б., Макаров М.В., СмирноваЕ.В. Казанский (Приволжский) федеральный университет Казань, Россия

doi: 10.18411/lj-31-03-2017-2-01

idsp 000001:lj-31-03-2017-2-01

\section{О псевдомонотонности оператора геометрически нелинейной задачи об изгибе трехслойной пластины}

\section{Введение.}

Настоящая работа посвящена изучению геометрически нелинейной задачи об изгибе трехслойной пластины с трансверсально-мягким заполнителем. Как отмечается в [1], многослойные конструкции находят все большее применение в различных отраслях народного хозяйства. Благодаря целесообразному выбору и составу отдельных слоев могут быть созданы многослойные панели с отличными статическими и конструктивными свойствами. Идея применения композитных материалов не нова, одно из первых упоминаний об использовании их можно найти в [2] (Исход, гл. 5), но благодаря авиации и полетам в космос, когда требовались легкие и высокопрочные материалы, был дан толчок к интенсивному развитию облегченных конструкций. Области применения композиционных материалов не ограничены. Они применяются в авиации для высоконагруженных деталей самолетов (обшивки, лонжеронов, нервюр, панелей и т. д.) и двигателей (лопаток компрессора и турбины и т. д.), в космической технике для узлов силовых конструкций аппаратов, подвергающихся нагреву, для элементов жесткости, панелей, в автомобилестроении для облегчения кузовов, рессор, рам, панелей кузовов, бамперов и т. Д., в горной промышленности (буровой инструмент, детали комбайнов и т. д.), в гражданском строительстве (пролеты мостов, элементы сборных конструкций высотных сооружений и т. д.) и в других областях народного хозяйства. Применение композиционных материалов обеспечивает новый качественный скачок в увеличении мощности двигателей, энергетических и транспортных установок, уменьшении массы машин и приборов. В трехслойных конструкциях нормальные усилия при изгибе воспринимаются обшивками, а сдвигающие - обрамлением или средним слоем. Средний слой обеспечивает также функции изоляции, восприятие местных нагрузок и устойчивость сжатых обшивок. Наиболее рациональное использование в трехслойных панелях свойств составляющих элементов делает эти панели предельно легкими и вместе с тем достаточно прочными, придавая им требуемые тепло-, звуко - и гидроизоляци-онные свойства [3-6].

Данная работа является продолжением [7-9] и посвящена доказательству 
псевдомонотонности [10, 11] введенного в [9] оператора геометрически нелинейной задачи об изгибе трехслойной пластины с трансверсально-мягким заполнителем, сформулированной в виде уравнения в пространстве Соболева. Обобщенные постановки для физически нелинейных и геометрически линейных задач в виде седловых задач, а также методы их решения рассмотрены в [12-16]. Физически нелинейные задачи теории мягких сетчатых оболочек изучены в [1725]. Численное решение геометрически нелинейных задач проводилось в [26-32].

\section{1. Постановка задачи.}

Рассматриваются задачи об определении напряженно-деформированного состояния бесконечно широкой трехслойной пластины с трансверсально-мягким заполнителем. Длина пластины равна $a$, толщина заполнителя составляет $2 \mathrm{t}$, толщины несущих слоев равны $2 t_{(k)}$, где $\mathrm{k}-$ номер слоя. Для описания напряженно-деформированного состояния (НДС) в несущих слояхиспользуются уравнения модели Кирхгофа-Лява, в заполнителе - уравнения теории упругости, упрощенные в рамках принятой моделитрансверсально-мягкого слоя и проинтегрированных по толщине судовлетворением условий сопряжения слоев по перемещениям $[33,34]$. В соответствии с $[33,34]$ введем следующие обозначения: $H_{(k)}=t+t_{(k)}$ (всюду в дальнейшем полагаем, что $\left.\mathrm{k}=1,2\right),{ }_{(k)}^{1}, X_{(k)}^{3}-$ компоненты поверхностной нагрузки, приведенной к срединной поверхности k-го слоя, $w^{(k)}$ и $u^{(k)}$ - прогибы и осевые перемещения точек срединной поверхности k-го слоя, $T_{(k)}^{11}, M_{(k)}^{11}$ - мембранные усилия и внутренние изгибающие моменты в k-м слое.Края пластины предполагаем закрепленными, т.е. ${ }^{(k)}(x)=0, w^{(k)}(x)=0$, $d w^{(k)}(x) / d x=0$ при $x=0, \quad x=a . \quad$ Рассматриваем $\quad$ геометрически нелинейныйслучай: $M_{(k)}^{11}=D_{(k)} d^{2} u^{(k)} / d x^{2}$, $T_{(k)}^{11}=B_{(k)}\left(d u^{(k)} / d x+0.5\left(d w^{(k)} / d x\right)^{2}\right), \quad$ где $B_{(k)}=2 t_{(k)} E^{(k)} /\left(1-v_{12}^{(k)} v_{21}^{(k)}\right)$ жесткость k-го слоя на растяжение-сжатие, $E^{(k)}{ }^{\left({ }^{\prime}\right.}{ }_{12}^{(k)}, v_{21}^{(k)}-$ модуль упругости первого рода и коэффициенты Пуассона материала k-го несущего слоя, $D_{(k)}=B_{(k)} t_{(k)}^{2} / 3$ - изгибная жесткость k-го слоя.Пусть $U=\left(w^{(1)}, w^{(2)}, u^{(1)}, u^{(2)}\right)$ вектор перемещений точек срединной поверхности k-го слоя, $q^{1}$ - касательные напряжения в заполнителе. Для $q^{1}$ считаем, что выполнены граничные условия $q^{1}(0)=q^{1}(a)=0 . \mathrm{B}[33,34]$ для описания НДСтрехслойной пластины был построен функционал $L\left(U, q^{1}\right)=P\left(U, q^{1}\right)--A_{e}\left(U, q^{1}\right)-A_{q}\left(U, q^{1}\right)$, где $P\left(U, q^{1}\right)$ потенциальная энергия деформации, $A_{e}\left(U, q^{1}\right)$ - работа заданных внешних сил и моментов, $A_{q}\left(U, q^{1}\right)$ - работа неизвестных контактных касательных напряжений на соответствующих перемещениях. Пусть $G_{13}, E_{3}$ - модули поперечного сдвига и 
обжатия заполнителя, $\quad c_{1}=2 t / G_{13}, \quad c_{2}=t^{3} /\left(3 E_{3}\right), \quad c_{3}=E_{3} /(2 t), \quad M_{(k)}^{1} \quad$ поверхностный момент внешних сил, приведенный к срединной поверхности k-го слоя, Установлено [34], что решением задачи о равновесии трехслойной пластины являются стационарные точки функционала L. Полагаем, что все постоянные $a$, $G_{13}, E_{3}, t, E^{(k)}, v_{12}^{(k)}, v_{21}^{(k)}, t_{(k)}, \mathrm{k}=1,2$, положительны.

\section{2. Операторная постановка задачи.}

Пусть $V_{k}=\stackrel{o}{W_{2}^{(k)}}(0, a) \_$пространства Соболева [35] со скалярными

произведениями

$$
(u, \eta)_{k}=\int_{0}^{a} d^{k} u / d x^{k} d^{k} \eta / d x^{k} d x
$$

Будем обозначать скалярное произведение в Vчерез $(\cdot,)_{V}$. В $[8,9]$ были полученыуравнения для стационарных точек функционалаLпутем вычисления производных Гато [36] этого функционала. Было установлено, что стационарные точки $\left(U, q^{1}\right)$ являются решением вариационного уравнения

$$
b\left(\left(U, q^{1}\right),(Z, y)\right)=f(Z) \quad \forall(Z, y) \in W=V \times V_{1},
$$

где форма $b(\cdot$,$) , заданная на W \times W$, и функционал $f$, заданный на $V$, определяются по формулам

$$
\begin{gathered}
b\left(\left(U, q^{1}\right),(Z, y)\right)=\int_{0}^{a} \sum_{k=1}^{2} B_{(k)}\left[\frac{d u^{(k)}}{d x}+\frac{1}{2}\left(\frac{d w^{(k)}}{d x}\right)^{2}\right] \frac{d \eta^{(k)}}{d x} d x+ \\
+\int_{0}^{a} \sum_{k=1}^{2} B_{(k)}\left[\frac{d u^{(k)}}{d x}+\frac{1}{2}\left(\frac{d w^{(k)}}{d x}\right)^{2}\right] \frac{d w^{(k)}}{d x} \frac{d z^{(k)}}{d x} d x+\int_{0}^{a} \sum_{k=1}^{2} D_{(k)} \frac{d^{2} w^{(k)}}{d x^{2}} \frac{d^{2} z^{(k)}}{d x^{2}} d x+ \\
+c_{3} \int_{0}^{a}\left(w^{(2)}-w^{(1)}\right)\left(z^{(2)}-z^{(1)}\right) d x+\int_{0}^{a}\left\{\sum_{k=1}^{2} H_{(k)} \frac{d z^{(k)}}{d x}+\left(\eta^{(2)}-\eta^{(1)}\right)\right\} q^{1} d x+(2) \\
+\int_{0}^{a}\left\{\left[\sum_{k=1}^{2} H_{(k)} \frac{d w^{(k)}}{d x}+\left(u^{(2)}-u^{(1)}\right)++c_{1} q^{1}\right] y+c_{2} d q^{1} / d x d y / d x\right\} d x=0 \\
\forall Z=\left(z^{(1)}, z^{(2)}, \eta^{(1)}, \eta^{(2)}\right) \in V, \quad \forall y \in V_{1}, \quad \forall Z \in V \\
f(Z)=\int_{0}^{a} \sum_{k=1}^{2}\left[X_{(k)}^{1} \eta^{(k)}+M_{(k)}^{1} \frac{d z^{(k)}}{d x}+X_{(k)}^{3} z^{(k)}\right] d x \quad \text { (3) }
\end{gathered}
$$

В [9] установлено, что форма $b(\cdot, \cdot)$, задаваемая (2), порождает оператор $A: W \rightarrow W$, определяемый формулой

$$
b\left(\left(U, q^{1}\right),(Z, y)\right)=\left(A\left(U, q^{1}\right),(Z, y)\right)_{W} \quad \forall(Z, y) \in W,
$$


где $(\cdot,)_{W}$ - скалярное произведение в $\mathrm{W}$, а функционал $\mathrm{f}$, задаваемый (3), порождает элемент $F \in V$, определяемый формулой $(F, Z)_{V}=f(Z)$ для всех $Z \in V$.

Кроме того, из полученных в [9] оценок с учетом очевидных неравенств $\left\|z^{(k)}\right\|_{2,2}=\left\|z^{(k)}\right\|_{2} \leq\|(Z, y)\|_{W}=\left(\left\|z^{(1)}\right\|_{2}^{2}+\left\|z^{(2)}\right\|_{2}^{2}+\left\|\eta^{(1)}\right\|_{1}^{2}+\left\|\eta^{(2)}\right\|_{1}^{2}+\|y\|_{1}^{2}\right)^{1 / 2}$ $\left\|\eta^{(k)}\right\|_{1,2}=\left\|\eta^{(k)}\right\|_{1} \leq\|(Z, y)\|_{W},\|y\|_{1,2}=\|y\|_{1} \leq\|(Z, y)\|_{W}$, справедливых для всех $Z=\left(z^{(1)}, z^{(2)}, \eta^{(1)}, \eta^{(2)}\right) \in V, y \in V$, вытекает, что

$\left|\left(A\left(U, q^{1}\right),(Z, y)\right)_{W}\right| \leq \alpha_{1}\left\|\left(U, q^{1}\right)\right\|_{W}\left(1+\left\|\left(U, q^{1}\right)\right\|_{W}+\left\|\left(U, q^{1}\right)\right\|_{W}^{2}\right)\|(Z, y)\|_{W}$, откуда следует, что справедлива

Лемма 1. Оператор $A: W \rightarrow W$, определяемый соотношениями (2), (3), является ограниченным.

Итак, задача (1) может быть записана в виде операторного уравнения

$$
A\left(U, q^{1}\right)=(F, 0)
$$

\section{3. Псевдомонотонность оператора А.}

Напомним, что оператор $A: Y \rightarrow Y$ называется псевдомонотонным[10, 11] если он ограничен и для любой слабо сходящейся последовательности $\left\{v_{k}\right\}_{k=1}^{+\infty} Y$ $v_{*} \quad \limsup \left(A v_{k}, v_{k}-v^{*}\right)_{Y} \leq 0$ к $v^{*}$ из неравенства ${ }_{k \rightarrow+\infty}$ для всех $\zeta$ из $Y$ вытекает, что $\liminf _{k \rightarrow+\infty}\left(A v_{k}, v_{k}-\zeta\right)_{Y} \geq\left(A v^{*}, v^{*}-\zeta\right)_{Y}$

При доказательстве псевдомонотонности оператора Анам потребуются следующие результаты. Справедлива теорема вложения Соболева [35, стр. 68].

Теорема 1. Пусть $\Omega \subset R^{n}$ - ограниченная область с регулярной границей $\Gamma$ и $1 \leq p<\infty$. Тогда $W_{p}^{(k)}(\Omega) \subset W_{r}^{(j)}(\Omega)$ для $0 \leq j<k$ и каждого $r$, удовлетворяющего условию $1 / p-(k-j) / n \leq 1 / r<1$; кроме того, для любой функции $u \in W_{p}^{(k)}(\Omega)$ справедливо неравенство

$$
\|u\|_{j, r} \leq C_{j r}^{k p}\|u\|_{k, p},
$$

где $\|\cdot\|_{k, p}$ - норма в $W_{p}^{(k)}(\Omega)$, а постоянная $C_{j r}^{k p}$ зависит от $\Omega, j, k, p, r$.

Далее, имеет место теорема Реллиха-Кондрашова о компактности вложений соболевских пространств [35, стр. 144].

Теорема 2. Пусть $\Omega \subset R^{n}$ - ограниченная область с регулярной границей $\Gamma$ и $1 \leq p<\infty$. Тогда $W_{p}^{(m+j)}(\Omega)$ компактно вкладывается в $W_{q}^{(j)}(\Omega)$ при выполнении условия $m p>n_{\text {для всех }} j \geq 0,1 \leq q<\infty$.

Отметим, что из компактности вложения X в Y следует сильная сходимость (по норме) в $\mathrm{X}$ последовательности, слабо сходящейся в Y. 
Теорема 3.Оператор $A: W \rightarrow W$, определяемый соотношениями (2), (3), является псевдомонотонным. Далее,

Доказательство. Ограниченность оператора А установлена в лемме 1.

$$
\begin{aligned}
& \left(A\left(U, q^{1}\right),\left(U, q^{1}\right)\right)_{W}=\int_{0}^{a} \sum_{k=1}^{2} B_{(k)}\left[\frac{d u^{(k)}}{d x}\right]^{2} d x+\int_{0}^{a} \sum_{k=1}^{2} D_{(k)}\left[\frac{d^{2} w^{(k)}}{d x^{2}}\right]^{2} d x+c_{2} \int_{0}^{a}\left[\frac{d q^{1}}{d x}\right]^{2} d x+ \\
& +\frac{3}{2} \int_{0}^{a} \sum_{k=1}^{2} B_{(k)} \frac{d u^{(k)}}{d x}\left[\frac{d w^{(k)}}{d x}\right]^{2} d x+\frac{3}{2} \int_{0}^{a} \sum_{k=1}^{2} B_{(k)}\left[\frac{d w^{(k)}}{d x}\right]^{4} d x+c_{3} \int_{0}^{a}\left(w^{(2)}-w^{(1)}\right)^{2} d x+ \\
& +\frac{1}{2} \int_{0}^{a} \sum_{k=1}^{2} H_{(k)} \frac{d w^{(k)}}{d x} q^{1} d x+2 \int_{0}^{a}\left(u^{(2)}-u^{(1)}\right) q^{1} d x+2 \int_{0}^{a}\left(q^{1}\right)^{2} d x=\sum_{j=1}^{9} I_{j}\left(U, q^{1}\right) . \\
& \text { Слабую сходимость будем обозначать символом } \stackrel{w}{\longrightarrow} \text {. Пусть } \\
& \left(U_{n}, q_{n}^{1}\right) \stackrel{w}{\longrightarrow}\left(U, q^{1}\right) \text { в W при } n \rightarrow+\infty \text {, т.е. } \\
& w_{n}^{(k)} \stackrel{w}{\longrightarrow} w^{(k)} \text { в } V_{2} \text { ив } L_{2}(0, a) \text { при } n \rightarrow+\infty \text {, } \\
& u_{n}^{(k)} \stackrel{w}{\longrightarrow} u^{(k)} \text { в } V_{1} \text { при } n \rightarrow+\infty \text {, } \\
& q_{n}^{1} \stackrel{w}{\longrightarrow} q^{1} \text { в } V_{1} \text { при } n \rightarrow+\infty .
\end{aligned}
$$

Из теоремы 2 и соотношения (6) вытекает, что

$$
w_{n}^{(k)} \rightarrow w^{(k)} \text { в } W_{q}^{(1)}(0, a) \text { и в } L_{q}(0, a), q \geq 1, \text { при } n \rightarrow+\infty .
$$

Из теоремы 2 и соотношений (7), (8) вытекает, что

$$
u_{n}^{(k)} \rightarrow u^{(k)}, q_{n}^{1} \rightarrow q \text { в } L_{q}(0, a), q \geq 1, \text { при } n \rightarrow+\infty .
$$

Кроме того, из (7)-(9) следует, что

$$
d w_{n}^{(k)} / d x \rightarrow d w^{(k)} / d x, d u_{n}^{(k)} / d x \stackrel{w}{\longrightarrow} d u^{(k)} / d x \text { в } L_{2}(0, a) \text { при } n \rightarrow+\infty \text {. }
$$

Далее (см., напр., [37]),

Если $v_{n} \rightarrow v^{*}, \zeta_{n} \stackrel{w}{\longrightarrow} \zeta^{*}$ в Ү при $n \rightarrow+\infty$, то $\left(v_{n}, \zeta_{n}\right)_{Y} \rightarrow\left(v^{*}, \zeta^{*}\right)_{Y}$ при $n \rightarrow+\infty$. (12)

В силу слабой полунепрерывности снизу [38] нормы (см., напр., [37, 39])имеем, что

$$
\liminf _{n \rightarrow+\infty} I_{j}\left(U_{n}, q_{n}^{1}\right) \geq I_{j}\left(U, q^{1}\right), \quad j=1,2,3 .
$$

Из (9) имеем, что $w_{n}^{(k)} \rightarrow w^{(k)}{ }_{\text {в }} W_{4}^{(1)}(0, a)$ при $n \rightarrow+\infty$, следовательно, применяя неравенство Минковского [40], получаем 


$$
\begin{gathered}
\int_{0}^{a}\left[\left(d w_{n}^{(k)} / d x\right)^{2}-\left(d w^{(k)} / d x\right)^{2}\right]^{2} d x= \\
=\int_{0}^{a}\left[d w_{n}^{(k)} / d x+d w^{(k)} / d x\right]^{2}\left[d w_{n}^{(k)} / d x-d w^{(k)} / d x\right]^{2} d x \leq \\
\leq\left\{\int_{0}^{a}\left[d w_{n}^{(k)} / d x+d w^{(k)} / d x\right]^{4}\right\}^{1 / 2}\left\{\int_{0}^{a}\left[d w_{n}^{(k)} / d x-d w^{(k)} / d x\right]^{4}\right\}^{1 / 2} \leq \\
\leq\left[\left\|w_{n}^{(k)}\right\|_{1,4}+\left\|w^{(k)}\right\|_{1,4}\right]^{2}\left\|w_{n}^{(k)}-w^{(k)}\right\|_{1,4}^{2} \rightarrow 0 \text { при } n \rightarrow+\infty,
\end{gathered}
$$

откуда

$$
\lim _{n \rightarrow+\infty} \int_{0}^{a}\left[\left(d w_{n}^{(k)} / d x\right)^{2}-\left(d w^{(k)} / d x\right)^{2}\right]^{2} d x=0
$$

и, с учетом (11), (12),

$$
\lim _{n \rightarrow+\infty} I_{4}\left(U_{n}, q_{n}^{1}\right)=I_{4}\left(U, q^{1}\right) .
$$

Принимая во внимание (14), получаем

$$
\begin{gathered}
\int_{0}^{a}\left[\left(d w_{n}^{(k)} / d x\right)^{4}-\left(d w^{(k)} / d x\right)^{4}\right] d x= \\
=\int_{0}^{a}\left[\left(d w_{n}^{(k)} / d x\right)^{2}+\left(d w^{(k)} / d x\right)^{2}\right]\left[\left(d w_{n}^{(k)} / d x\right)^{2}-\left(d w^{(k)} / d x\right)^{2}\right] d x \leq \\
\leq\left\{\int_{0}^{a}\left[\left(d w_{n}^{(k)} / d x\right)^{2}+\left(d w^{(k)} / d x\right)^{2}\right]^{2} d x\right\}^{1 / 2}\left\{\int_{0}^{a}\left[\left(d w_{n}^{(k)} / d x\right)^{2}-\left(d w^{(k)} / d x\right)^{2}\right]^{2} d x\right\}^{1 / 2} \leq \\
\leq\left[\left\|w_{n}^{(k)}\right\|_{1,4}^{2}+\left\|w^{(k)}\right\|_{1,4}^{2}\right]\left\{\int_{0}^{a}\left[\left(d w_{n}^{(k)} / d x\right)^{2}-\left(d w^{(k)} / d x\right)^{2}\right]^{2} d x\right\}^{1 / 2} \rightarrow 0 \quad \text { при } n \rightarrow+\infty,
\end{gathered}
$$

а значит,

$$
\lim _{n \rightarrow+\infty} I_{5}\left(U_{n}, q_{n}^{1}\right)=I_{5}\left(U, q^{1}\right) .
$$

Наконец, из (9)-(11) и непрерывности нормы вытекает, что

$$
\lim _{n \rightarrow+\infty} I_{j}\left(U_{n}, q_{n}^{1}\right)=I_{j}\left(U, q^{1}\right), \quad j=6,7,8,9 . .
$$


Используя теперь то, что если $\liminf _{k \rightarrow+\infty} \alpha_{k} \geq \alpha \quad \lim _{k \rightarrow+\infty} \beta_{k}=\beta$, то $\liminf _{k \rightarrow+\infty}\left(\alpha_{k}+\beta_{k}\right) \geq \alpha+\beta$

$$
\begin{array}{r}
\text { (см., напр. [41]), из (13), (15)-(17) получаем } \\
\liminf _{n \rightarrow+\infty}\left(A\left(U_{n}, q_{n}^{1}\right),\left(U_{n}, q_{n}^{1}\right)\right)_{W} \geq\left(A\left(U, q^{1}\right),\left(U, q^{1}\right)\right)_{W} .
\end{array}
$$

Из (11) следует, что

$$
\begin{gathered}
\lim _{n \rightarrow+\infty} \int_{0}^{a}\left[\frac{d u_{n}^{(k)}}{d x}-\frac{d u^{(k)}}{d x}\right] \frac{d \eta^{(k)}}{d x} d x=0, \quad \lim _{n \rightarrow+\infty} \int_{0}^{a}\left[\frac{d q_{n}^{1}}{d x}-\frac{d q^{1}}{d x}\right] \frac{d y}{d x} d x=0, \\
\lim _{n \rightarrow+\infty} \int_{0}^{a}\left[\frac{d w_{n}^{(k)}}{d x}-\frac{d w^{(k)}}{d x}\right] y d x=0 .
\end{gathered}
$$

Из (14) следует, что

$$
\lim _{n \rightarrow+\infty} \int_{0}^{a}\left[\left(d w_{n}^{(k)} / d x\right)^{2}-\left(d w^{(k)} / d x\right)^{2}\right] d \eta^{(k)} d x=0 .
$$

Из (9) следует, что

$$
\lim _{n \rightarrow+\infty} \int_{0}^{a}\left[\left(w_{n}^{(2)}-w_{n}^{(1)}\right)-\left(w^{(2)}-w^{(1)}\right)\right]\left(z^{(2)}-z^{(1)}\right) d x=0 .
$$

Из (10) следует, что

$$
\begin{aligned}
& \lim _{n \rightarrow+\infty} \int_{0}^{a}\left[\left(u_{n}^{(2)}-u_{n}^{(1)}\right)-\left(u^{(2)}-u^{(1)}\right)\right] y d x=0, \\
& \lim _{n \rightarrow+\infty} \int_{0}^{a}\left(q_{n}^{1}-q^{1}\right)\left[d z^{(k)} / d x+\left(\eta^{(2)}-\eta^{(1)}\right)\right] d x=0 .
\end{aligned}
$$

Далее, в силу теоремы $1 w^{(k)} \in W_{q}^{(1)}(0, a), w_{n}^{(k)} \in W_{q}^{(1)}(0, a)$ для любого $q \geq 1$ , а значит, в силу обобщенного неравенства Гельдера с показателями 2, 4, 4 [40]

$$
\begin{gathered}
\int_{0}^{a}\left[\frac{d u_{n}^{(k)}}{d x}\left(\frac{d w_{n}^{(k)}}{d x}\right)^{3}-\frac{d u^{(k)}}{d x}\left(\frac{d w^{(k)}}{d x}\right)^{3}\right] \frac{d z^{(k)}}{d x} d x= \\
=\int_{0}^{a} \frac{d u_{n}^{(k)}}{d x}\left(\left(\frac{d w_{n}^{(k)}}{d x}\right)^{3}-\left(\frac{d w^{(k)}}{d x}\right)^{3}\right) \frac{d z^{(k)}}{d x}+\int_{0}^{a}\left(\frac{d u_{n}^{(k)}}{d x}-\frac{d u^{(k)}}{d x}\right)\left(\frac{d w^{(k)}}{d x}\right)^{3} \frac{d z^{(k)}}{d x} d x \leq
\end{gathered}
$$




$$
\begin{aligned}
\leq\left(\int_{0}^{a}\left(\frac{d u_{n}^{(k)}}{d x}\right)^{2}\right)^{1 / 2} & \left(\int_{0}^{a}\left(\left(\frac{d w_{n}^{(k)}}{d x}\right)^{3}-\left(\frac{d w^{(k)}}{d x}\right)^{3}\right)^{4} d x\right)^{1 / 4}\left(\int_{0}^{a}\left(\frac{d z^{(k)}}{d x}\right)^{4} d x\right)^{1 / 4}+ \\
& +\int_{0}^{a}\left(\frac{d u_{n}^{(k)}}{d x}-\frac{d u^{(k)}}{d x}\right)\left(\frac{d w^{(k)}}{d x}\right)^{3} \frac{d z^{(k)}}{d x} d x
\end{aligned}
$$

Слабо сходящаяся последовательность ограничена: $\left(\int_{0}^{a}\left(\frac{d u_{n}^{(k)}}{d x}\right)^{2}\right)^{1 / 2} \leq \alpha_{3}$, в силу теоремы $1 z^{(k)} \in W_{4}^{(1)}(0, a)$.Наконец,

$$
\begin{aligned}
& \left(\int_{0}^{a}\left(\left(\frac{d w_{n}^{(k)}}{d x}\right)^{3}-\left(\frac{d w^{(k)}}{d x}\right)^{3}\right)^{4} d x\right)^{1 / 4} \leq \\
& \leq\left\|w_{n}^{(k)}-w^{(k)}\right\|_{1,16}\left(\left\|w_{n}^{(k)}\right\|_{1,8}+\left\|w^{(k)}\right\|_{1,8}+\left\|w_{n}^{(k)}\right\|_{1,16}\left\|w^{(k)}\right\|_{1,16}\right) \leq \\
& \leq\left\|w_{n}^{(k)}-w^{(k)}\right\|_{1,16}\left(\alpha_{4}+\alpha_{5}\left\|w^{(k)}\right\|_{1,16}+\left\|w^{(k)}\right\|_{1,8}\right) \rightarrow 0 \quad \text { при } n \rightarrow+\infty,
\end{aligned}
$$

а значит,

$$
\lim _{n \rightarrow+\infty} \int_{0}^{a}\left[\frac{d u_{n}^{(k)}}{d x}\left(\frac{d w_{n}^{(k)}}{d x}\right)^{3}-\frac{d u^{(k)}}{d x}\left(\frac{d w^{(k)}}{d x}\right)^{3}\right] \frac{d z^{(k)}}{d x} d x=0 .
$$

$$
\liminf _{n \rightarrow+\infty}\left(A\left(U_{n}, q_{n}^{1}\right),(Z, y)\right)_{W}=\left(A\left(U, q^{1}\right),(Z, y)\right)_{W}
$$

Из (19)-(23) вытекает, что $n \rightarrow+\infty$

для любого $(Z, y) \in W$, откуда с учетом (18) и вытекает псевдомонотонность оператора A.

\section{4. Заключение.}

Доказано, что оператор, возникающий при математическом описании геометрически нелинейной задачи об изгибе трехслойной пластины с трансверсально-мягким заполнителем является псевдомонотонным. Это дает возможность использовать при исследовании корректности задачи (1) по аналогии c [42-49] использовать результаты общей теории монотонных операторов. Кроме того данное свойство может быть полезным при исследовании сходимости приближенных методов решения задачи. При этом будут использованы разработанные в [50-61] подходы.

Публикаиия осуществлена при финансовой поддержке РФФИ (проекты №№ 15-01-05686, 16-38-00788, 17-08-01279). 


\section{Литература}

1. Штамм К., Витте Х. Многослойные конструкции. - М.: Стройиздат, 1983. - 300 с.

2. Библия. Книги священного писания Ветхого и Нового Завета. - М.: Московская Патриархия, 1988. - 1371 с.

3. Макаров М.В., Бадриев И.Б., Паймушин В.Н. Нелинейные задачи о смешанных формах потери устойчивости трехслойных пластин при продольно-поперечном изгибе // Вестник Тамбовского университета. Серия: Естественные и технические науки. - 2015. - Т. 20, № 5. - С. 1275-1278.

4. Бадриев И.Б., Бандеров В.В., Макаров М.В., Паймушин В.Н. Решение нелинейных задач теории многослойных оболочек с трансверсально-мягким заполнителем // В сборнике: Сеточные методы для краевых задач и приложения Материалы Десятой Международной конференции, 2014. - С. 103-107.

5. Paimushin V.N. Problems of geometric non-linearity and stability in the mechanics of thin shells and rectilinear columns // Journal of Applied Mathematics and Mechanics. 2007. - V. 71, № 5. - P. 772-805.

6. Бадриев И.Б., Макаров М.В., Паймушин В.Н. Контактная постановка задач механики подкрепленных на контуре трехслойных оболочек с трансверсальномягким заполнителем // Известия высших учебных заведений. Математика. - 2017. - № 1. - С. 77-85.

7. Бадриев И.Б., Макаров М.В., Мартынова О.П. Постановка геометрически нелинейной задачи об изгибе трехслойной пластины с трансверсально-мягким заполнителем // Тенденции науки и образования в современном мире. - 2016. - № 16-1. - С. 5-11.

8. Бадриев И.Б., Макаров М.В., Мартынова О.П. Операторная формулировка геометрически нелинейной задачи об изгибе трехслойной пластины с трансверсально-мягким заполнителем // Тенденции науки и образования в современном мире. - 2016. - № 18-3. - С. 5-13.

9. Бадриев И.Б., Макаров М.В., Смирнова Е.В. Обобщенная постановка геометрически нелинейной задачи об изгибе трехслойной пластины // Тенденции науки и образования в современном мире. - 2017. - № 23-1. - С. 4-10.

10. Brezis H. Équations et inéquations non-linéairesdans les espacesvectoriels en dualité // Annales de l'institut Fourier (Grenoble). - 1968. - V. 19. - P. 115-175.

11. Лионс Ж.-Л. Некоторые методы решения нелинейных краевых задач. - М.: Мир, 1972. $-588 \mathrm{c}$.

12. Бадриев И.Б., Бандеров В.В., Макаров М.В. Численное исследование физически нелинейных задач об изгибе трехслойных пластин // В сборнике: Материалы XI Международной конференции по неравновесным процессам в соплах и струях (NPNJ'2016) Московский авиационный институт (национальный исследовательский университет), 2016. - С. 475-477.

13. Бадриев И.Б., Гарипова Г.З., Макаров М.В., Паймушин В.Н., Хабибуллин Р.Ф. О решении физически нелинейных задач о равновесии трехслойных пластин с трансверсально-мягким заполнителем // Ученые записки Казанского университета. Серия: Физико-математические науки. - 2015. - Т. 157, № 1. - С. 15-24.

14. Бадриев И.Б., Бандеров В.В., Гарипова Г.З., Макаров М.В. О разрешимости нелинейной задачи о равновесии трехслойной пластины // Вестник Тамбовского 
университета. Серия: Естественныеитехническиенауки. - 2015. - Т. 20, № 5. - С. 1034-1037.

15. Бадриев И.Б., Гарипова Г.З., Макаров М.В. Сеточные аппроксимации физически нелинейной задачи об изгибе трехслойной пластины с трансверсально-мягким заполнителем // В сборнике: Воронежская зимняя математическая школа С.Г. Крейна - 2016. Материалы международной конференции. - Воронеж: Издательскополиграфический центр «Научная книга», 2016. - С. 70-72.

16. Badriev I.B., Garipova G.Z., Makarov M.V., Paimushin V.N., Khabibullin R.F. Solving physically nonlinear equilibrium problems for sandwich plates with a transversally soft core // Lobachevskii Journal of Mathematics. - 2015. - V. 36, № 4. - P. 474-481.

17. Badriev I.B., Banderov V.V. Numerical solution of the equilibrium of axisymmetric soft shells // Математическиеметоды в технике и технологиях - ММТТ. - 2014. - № 5 (64). - C. 16-20.

18. Badriev I.B., Banderov V.V., Gnedenkova V.L., Kalacheva N.V., Korablev A.I., Tagirov R.R. On the finite dimensional approximations of some mixed variational inequalities // Applied Mathematical Sciences. - 2015. - V. 9, № 113-116. - P. 5697-5705.

19. Бадриев И.Б. Решение вариационных задач нелинейных процессов механики сплошной среды // В книге: Теория управления и математическое моделирование Тезисы докладов Всероссийской конференции с международным участием, посвященной памяти профессора Н.В. Азбелева и профессора Е.Л. Тонкова. Удмуртский государственный университет. - Ижевск: Изд-во "Удмуртский университет", 2015. - С. 234-235.

20. Бадриев И.Б. О решении вариационных неравенств второго рода // В сборнике: Сеточные методы для краевых задач и приложения Материалы Девятой Всероссийской конференции. - Казань: Отечество, 2012. - С. 32-35.

21. Бадриев И.Б., Бандеров В.В. Численное моделирование деформирования биокомпозитов // В книге: Динамические и технологические проблемы механики конструкций и сплошных сред Материалы XXI международного симпозиума имени А.Г. Горшкова. Московский авиационный институт (национальный исследовательский университет). - Москва: ООО "ТРП", 2015. - С. 7-9.

22. Бадриев И.Б., Задворнов О.А. Исследование разрешимости осесимметричной задачи об определении положения равновесия мягкой оболочки вращения // Известия высших учебных заведений. Математика. - 2005. - № 1. - С. 25-30.

23. Бадриев И.Б., Бандеров В.В., Калачева Н.В. Численное исследование вариационных и квазивариационных неравенств теории мягких сетчатых оболочек // Вестник Тамбовского университета. Серия: Естественные и технические науки. - 2015. - Т. 20, № 5. - С. 1037-1041.

24. Бадриев И.Б., Бандеров В.В. Численное решение задач о равновесии осесимметричных мягких оболочек // Вестник Тамбовского государственного технического университета. - 2015. - Т. 21, № 1. - С. 29-35.

25. Бадриев И.Б., Бандеров В.В. Итерационные методы решения вариационных неравенств теории мягких оболочек // Ученые записки Казанского университета. Серия: Физико-математические науки. - 2013. - Т. 155, кн.2. - С. 18-32.

26. Badriev I.B., Garipova G.Z., Paymushin V.N., Makarov M.V. Numerical solution of the issue about geometrically nonlinear behavior of sandwich plate with transversal soft filler // Research Journal of Applied Sciences. - 2015. - V. 10, № 8. - P. 428-435.

27. Бадриев И.Б., Желтухин В.С., Чебакова В.Ю. О решении некоторых нелинейных краевых и начально-краевых задач // В книге: Материалы XXII Международного симпозиума «Динамические и технологические проблемы механики конструкций и сплошных сред» им. А.Г. Горшкова Московский авиационный институт 
(национальный исследовательский университет). - Москва: ООО "ТРП", 2016. - С. 31-33.

28. Бадриев И.Б., Макаров М.В., Паймушин В.Н. Разрешимость физически и геометрически нелинейной задачи теории трехслойных пластин с трансверсальномягким заполнителем // Известия высших учебных заведений. Математика. - 2015. - № 10. - С. 66-71.

29. Макаров М.В., Бадриев И.Б., Паймушин В.Н. Определение критических точек бифуркации для задачи о геометрически нелинейном поведении трехслойной пластины с трансверсально-мягким заполнителем // В сборнике: Воронежская зимняя математическая школа С. Г. Крейна - 2016. Материалы международной конференции. - Воронеж: Издательско-полиграфический центр «Научная книга», 2016. - С. 292-294.

30. Бадриев И.Б., Бандеров В.В., Макаров М.В., Паймушин В.Н. Исследование задачи об изгибе трехслойной пластины в физически нелинейной постановке // В сборнике: Математическое моделирование и краевые задачи Труды десятой Всероссийской научной конференции с международным участием: в 3-х томах, 2016. - C. 19-24.

31. Макаров М.В. Численное исследование геометрически нелинейной задачи о поперечном изгибе трехслойной пластины с трансверсально-мягким заполнителем // Тенденции науки и образования в современном мире. - 2016. -№ 17-2. - С. 26-35.

32. Макаров М.В. О решении геометрически нелинейных и линейных задач о поперечном изгибе жестко закрепленной трехслойной пластины с трансверсальномягким заполнителем // Тенденции науки и образования в современном мире. 2016. - № 19-2. - C. 23-29.

33. Paimushin V.N., Bobrov S.N. Refined geometric nonlinear theory of sandwich shells with a transversely soft core of medium thickness for investigation of mixed buckling forms // Mechanics of Composite Materials. - 2000. - V. 36, № 1. - P. 59-66.

34. Паймушин В.Н. Обобщенный вариационный принцип Рейсснера в нелинейной механике пространственных составных тел с приложениями к теории многослойных оболочек // Известия Российской академии наук. Механикатвердоготела. - 1987. - № 2. - С. 171.

35. Adams R.A.Sobolev Spaces. - New York, San Francisco, London: Academic Press, 1975. -286 p.

36. Вайнберг М.М. Вариационный метод и метод монотонных операторов. - М: Наука, 1972. - $416 \mathrm{c}$.

37. Бадриев И.Б., Задворнов О.А. Итерационные методы решения вариационных неравенств в гильбертовых пространствах. - Казань: Казанский государственный университет, 2007. - 152 с.

38. EkelandI., Temam R. Convex Analysis and Variational Problems, Amsterdam: NorthHolland, 1976. - 402 p.

39. Васильев Ф.П. Методы решения экстремальных задач. - М.: Наука, 1981. - 400 с.

40. Hardy G.H., Littlewood J.E., Pólya G. Inequalities. - Cambridge: CambridgeUniversity Press, 1934. - 314 p.

41. Теляковский С.А. Курс лекций по математическому анализу. Семестр I. - М.: МИАН, 2009. - 212 с.

42. Бадриев И.Б. Математическое моделирование процессов механики сплошной среды при наличии точечных источников и нагрузок // В книге: Материалы XXII Международного симпозиума «Динамические и технологические проблемы механики конструкций и сплошных сред» им. А.Г. Горшкова Московский 
авиационный институт (национальный исследовательский университет). - Москва: ООО "ТРП", 2016. - С. 28-31.

43. Badriev I.B. Mathematicalmodelingofcontinuummechanicsproblemswiththepointsources // В сборнике: Проблемы химмотологии: от эксперимента к математическим моделям высокого уровня. Сборник избранных докладов VI Международной научно-технической конференции, 2016. - С. 19-21.

44. Бадриев И.Б., Фанюк Б.Я. Итерационные методы решения задач фильтрации в многослойных пластах при наличии точечного источника // Ученые записки Казанского университета. Серия: Физико-математические науки. - 2010. - Т. 152, № 4. - С. 39-55.

45. Бадриев И.Б., Сингатуллин М.Т., Чебаков Ю.В. Численное исследование задач подземной установившейся фильтрации высоковязких жидкостей при наличии скважин // Вестник Тамбовского университета. Серия: Естественныеитехническиенауки. - 2015. - Т. 20, № 5. - С. 1041-1044.

46. Badriev I.B., Banderov V.V., Singatullin M.T. Numerical solution of non-linear filtration issues for high viscous fluids at the presence of wells // Research Journal of Applied Sciences. - 2015. - V. 10, № 8. - P. 343-346.

47. Badriev I.B., Fanyuk B.Y. Iterative methods for solving seepage problems in multilayer beds in the presence of a point source // Lobachevskii Journal of Mathematics. - 2012. V. 33, № 4. - P. 386-399.

48. Бадриев И.Б., Задворнов О.А. Итерационные методы решения вариационных неравенств второго рода с обратно сильно монотонными операторами // Известия высших учебных заведений. Математика. - 2003. - № 1. - С. 20-28.

49. Желтухин В.С., Чебакова В.Ю., Шнейдер М.Н. Моделирование ВЧЕ-разряда при больших межэлектродных расстояниях // В сборнике: Сеточные методы для краевых задач и приложения. Материалы Девятой Всероссийской конференции. Казань: Отечество, 2012. - С. 183-186.

50. Бадриев И.Б., Задворнов О.А. О сходимости итерационного метода двойственного типа решения смешанных вариационных неравенств // Дифференциальные уравнения. - 2006. - Т. 42, № 8. - С. 1115-1122.

51. Абдуллин И.Ш., Желтухин В.С., Чебакова В.Ю., Шнейдер М.Н. Тематическая модель ВЧЕ-разряда в аргоне при больших межэлектродных расстояниях // Вестник Казанского технологического университета. - 2013. - Т. 16, № 22. - С. 334-338.

52. Бикчантаев Д.Х., Желтухин В.С., Чебакова В.Ю. Численное исследование процесса взаимодействия высокочастотного емкостного разряда с материалами // В книге: Теория управления и математическое моделирование. Тезисы докладов Всероссийской конференции с международным участием, посвященной памяти профессора Н.В. Азбелева и профессора Е.Л. Тонкова. Удмуртский государственный университет. - Ижевск: Изд-во "Удмуртский университет", 2015. - C. 241-242.

53. Желтухин В.С., Соловьёв С.И., Соловьёв П.С., Чебакова В.Ю. Вычисление минимального собственного значения нелинейной задачи Штурма - Лиувилля // Ученые записки Казанского университета. Серия: Физико-математические науки. 2013. - Т. 155, № 3. - С. 91-104.

54. Бадриев И.Б., Ляшко А.Д., Панкратова О.В. Исследование сходимости итерационных методов решения нелинейных задач теории фильтрации // Известия высших учебных заведений. Математика. - 1998. - № 11. - С. 8-13.

55. Бадриев И.Б. Математическое моделирование стационарных задач подземной фильтрации с многозначным законом // Вестник Тамбовского университета. Серия: Естественные и технические науки. - 2013. - Т. 18, № 5-2. - С. 2444-2446. 
56. Чебакова В.Ю. Моделирование высокочастотного емкостного разряда при атмосферном давлении в аргоне // Ученые записки Казанского университета. Серия: Физико-математические науки. - 2016. - Т. 158, № 3. - С. 404-423.

57. Бадриев И.Б., Нечаева Л.А. Математическое моделирование установившейся фильтрации с многозначным законом // Вестник Пермского национального исследовательского политехнического университета. Механика. - 2013. - № 3. - С. 35-62.

58. Бадриев И.Б., Исмагилов И.Н. Итерационные методы решения нелинейных стационарных задач анизотропной фильтрации // Журнал Средневолжского математического общества. - 2006. - Т. 8, № 1.- С. 150-159.

59. Badriev I.B., Makarov M.V., Paimushin V.N. Numerical investigation of physically nonlinear problem of sandwich plate bending // B сборнике: Procedia Engineering 2. Cep. "2nd International Conference on Industrial Engineering, ICIE 2016", 2016. - P. 1050-1055.

60. Badriev I.B., Makarov M.V., Paimushin V.N. Mathematical simulation of nonlinear problem of three-point composite sample bending test // B сборнике: Procedia Engineering 2. Cep. "2nd International Conference on Industrial Engineering, ICIE 2016", 2016. - P. 1056-1062.

61. Бадриев И.Б., Чебакова В.Ю. Математическое моделирование низкотемпературной ВЧЕ-плазмы в аргоне // В сборнике: Математическое моделирование и краевые задачи Труды десятой Всероссийской научной конференции с международным участием: в 3-х томах, 2016. - С. 17-21. 